\title{
Mobilization practices in critically ill children: a European point prevalence study (EU PARK-PICU)
}

\author{
Erwin Ista ${ }^{1,2^{*}}$ D, Barnaby R. Scholefield ${ }^{3,4}$, Joseph C. Manning ${ }^{5,6}$, Irene Harth , Orsola Gawronski ${ }^{8}$, \\ Alicja Bartkowska-Śniatkowska ${ }^{9}$, Anne-Sylvie Ramelet ${ }^{10}$, Sapna R. Kudchadkar ${ }^{11,12,13}$ and EU PARK-PICU \\ Collaborators $14,15,16,17,18,19,20,21,22,23,24,25,26,27,28,29,30,31,32,33,34,35,36,37,38,39,40,41,42,43,44,45,46,47,48$
}

\begin{abstract}
Background: Early mobilization of adults receiving intensive care improves health outcomes, yet little is known about mobilization practices in paediatric intensive care units (PICUs). We aimed to determine the prevalence of and factors associated with physical rehabilitation in PICUs across Europe.
\end{abstract}

Methods: A 2-day, cross-sectional, multicentre point prevalence study was conducted in May and November 2018. The primary outcome was the prevalence of physical therapy (PT)- or occupational therapy (OT)-provided mobility. Clinical data and data on patient mobility, potential mobility safety events, and mobilization barriers were prospectively collected in patients admitted for $\geq 72 \mathrm{~h}$.

Results: Data of 456 children admitted to one of 38 participating PICUs from 15 European countries were collected (456 patient days); $70 \%$ were under 3 years of age. The point prevalence of PT- and/or OT-provided mobility activities was 39\% (179/456) (95\% Cl 34.7-43.9\%) during the patient days, with significant differences between European regions. Nurses were involved in 72\% (924/1283) of the mobility events; in the remaining 28\%, PT/OT, physicians, family members, or other professionals were involved. Of the factors studied, family presence was most strongly positively associated with out-of-bed mobilization (aOR 7.83, 95\% Cl 3.09-19.79). Invasive mechanical ventilation with an endotracheal tube was negatively associated with out-of-bed mobility (aOR $0.28,95 \% \mathrm{Cl} 0.12-$ 0.68). Patients were completely immobile on $25 \%(115 / 456)$ of patient days. Barriers to mobilization were reported on $38 \%$ of patient days. The most common reported patient-related barriers were cardiovascular instability $(n=47$, $10 \%)$, oversedation $(n=39,9 \%)$, and medical contraindication $(n=37,8 \%)$. Potential safety events occurred in $6 \%$ of all documented mobilization events.

Conclusion: Therapists are infrequently consulted for mobilization of critically ill children in European PICUs. This study highlights the need for a systematic and interdisciplinary mobilization approach for critically ill children.

Keywords: Critical care, Paediatrics, Rehabilitation, Physical therapy, Occupational therapy, Developmental paediatrics, Intensive care units

\footnotetext{
*Correspondence: w.ista@erasmusmc.nl

${ }^{1}$ Pediatric Intensive Care Unit, Department of Paediatric Surgery, Erasmus

Medical Center - Sophia Children's Hospital, P.O. Box 2060, 3000 CB

Rotterdam, The Netherlands

${ }^{2}$ Nursing Science, Department of Internal Medicine, Erasmus Medical Center, Rotterdam, The Netherlands

Full list of author information is available at the end of the article
}

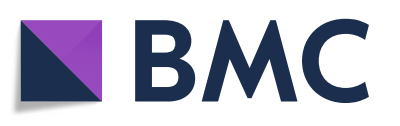

( ) The Author(s). 2020 Open Access This article is licensed under a Creative Commons Attribution 4.0 International License, which permits use, sharing, adaptation, distribution and reproduction in any medium or format, as long as you give appropriate credit to the original author(s) and the source, provide a link to the Creative Commons licence, and indicate if changes were made. The images or other third party material in this article are included in the article's Creative Commons licence, unless indicated otherwise in a credit line to the material. If material is not included in the article's Creative Commons licence and your intended use is not permitted by statutory regulation or exceeds the permitted use, you will need to obtain permission directly from the copyright holder. To view a copy of this licence, visit http://creativecommons.org/licenses/by/4.0/. The Creative Commons Public Domain Dedication waiver (http://creativecommons.org/publicdomain/zero/1.0/) applies to the data made available in this article, unless otherwise stated in a credit line to the data. 


\section{Background}

The paediatric intensive care unit is a stressful environment for critically ill children. To provide safety, comfort, and hemodynamic stability, children are often sedated and considered too sick to be mobilized [1]. However, immobility is associated with adverse effects such as muscle weakness [2, $3]$, pressure ulcers $[4,5]$, increased risk of a delirium [6], and post-intensive care syndrome [7-9]. Ideally, sedation is targeted to facilitate ICU procedures while keeping the patient comfortable without anxiety and agitation.

Early mobilization of patients in adult ICUs has proven feasible and safe with favourable clinical outcomes including decreased delirium incidence and duration of ICU stay, less sedatives consumption, and improved muscle strength and functional status [6, 10]. Early mobilization in critically ill children undergoing active neurocognitive and physical development is understudied. An early mobilization project in the USA targeting critically ill children showed promising results with no adverse events [11]; this interdisciplinary approach doubled the number of mobilization events per patient.

A recent point prevalence study of physical rehabilitation in United States PICUs (PARK-PICU: Prevalence of Acute Rehabilitation for Kids in the PICU) demonstrated that younger children and those with higher baseline function were less likely to receive therapist-provided mobility [12]. In view of the lack of similar data in European countries, we adapted the PARK-PICU study in Europe to determine the prevalence of routine mobilization of children admitted to a PICU for at least $72 \mathrm{~h}$. Additionally, we explored patient-related barriers and potential adverse events related to PICU mobilization.

\section{Methods}

The European Prevalence of Acute Rehabilitation for Kids in the PICU study (EU-PARK-PICU) was a 2-day crosssectional point prevalence study (May 29, 2018, and November 6, 2018). PICUs from European countries were eligible to participate if they met the following criteria: (1) provide care for mechanically ventilated infants and children and (2) located in a distinct physical space within a hospital dedicated to paediatric patients. Sites were recruited via the European Society of Paediatric and Neonatal Intensive Care (ESPNIC) network, e-mail, social media, and a dedicated website (https://park.web.jhu.edu). Institutional review board or clinical governance approval was obtained at all sites with waiver of informed consent. For comparability across studies internationally, we utilized the same methodology as the original PARK-PICU study in the United States of America (USA) [12].

\section{Patient selection}

Eligible patients were those who had been admitted to PICUs for $\geq 72 \mathrm{~h}$ as of $7 \mathrm{a} . \mathrm{m}$. on each of the two study days. This criterion was set because admission longer than 3 days carries greater risk for muscle atrophy and physical impairment [13], and adult ICU studies suggest this as the threshold for early rehabilitation and mobilization $[6,14,15]$.

\section{Notification, study day selection, and data collection}

An information brochure (available in English, Italian, French, German, Polish, Spanish, and Dutch) was provided to interested sites. Each participating site was informed about the designated months for the point prevalence once consent was provided. On the first day of the month, at random a weekday for the study was chosen to conduct the study in all PICUs. The local study teams were notified by email that they should screen for eligibility on that day and prepare for data collection on the following day, starting at 9 a.m. and continued until 9 a.m. the next day.

\section{Measures}

All data collection forms were adapted from those used in the USA PARK-PICU study. In addition to English, bedside data collection forms (e.g. Activity events) were translated to Dutch, French, Spain, German, and Italian are available on the study website [16].

\section{PICU characteristics}

Each participating PICU completed an electronic online survey (LimeSurvey@) to provide administrative data and information about clinical resources and protocols related to pain, sedation and delirium management, ventilator weaning, early mobilization, and family engagement. To ensure accuracy, site investigators were instructed to complete the questionnaire together with a physician and the PICU nurse manager.

\section{Patient clinical characteristics}

Sites extracted clinical data for all eligible patients, including clinical status at 9 a.m. on the point prevalence day: mechanical ventilation status, sedative infusions and level of sedation, delirium screening, and invasive catheters. Mechanical ventilation was defined as ventilation through an endotracheal tube, a tracheostomy, or a face/nasal mask. Pre-admission physical function categorized by the Paediatric Cerebral Performance Score (PCPC) was extracted from the medical record or, if missing, on the basis of information from family and care team $[16,17]$.

\section{Mobility data}

Physical therapy (PT) and occupational therapy (OT) consultation and treatment documentation for the first $72 \mathrm{~h}$ of PICU admission were abstracted from the electronic patient record or other records. Standardized forms with checklists were distributed to the bedside of 
each eligible patient by 9 a.m. for real-time event recording. Nurses and other healthcare staff were instructed to document the following: (a) occurrence of any mobility activity provided by a therapist, nurse, family, and/or other staff; (b) type (in-bed/out-of-bed) and timing of mobility events; (c) perceived barriers to mobilization; and (d) safety events associated with mobilization-e.g. a change of $\geq 10 \%$ in heart rate, oxygen saturation, or blood pressure; loss of invasive devices; and falls. Outof-bed mobility was defined as transfer from bed to chair, being held by family or staff, mat play, standing, or walking. Activities such as passive motion, sitting in bed, and bath were defined as in-bed mobility. Mobility events were defined as any single activity or clustered activities involving the child's physical movement, with the exception of routine care procedures and turning/repositioning or prevention of pressure ulcers. Each separate activity was recorded on a separate form. Both barriers and potential safety events were selected from a prespecified list with a free-text option.

The primary outcome was "therapist-provided mobility", defined as at least one mobility event performed by a PT or OT on the study day. This primary outcome measure was chosen for the following reasons: (1) comparability to US paediatric and adult point prevalence data [18] and (2) information from participating PICUs that PT and OTs are often consulted simultaneously and the rehabilitation team determines which services are most appropriate to provide. Secondary outcomes were out-of-bed mobility, barriers to mobilization, and potential safety events.

\section{Data analysis}

The prevalence of therapist-provided mobility was defined as the number of patient days with therapistprovided mobility divided by the total number of patient days across the two study days. Also, the prevalence of out-of-bed mobility was calculated as the number of patients with out-of-bed mobility provided by a healthcare professional (e.g. PT, OT, or nurse) divided by the total number of patient days across the study days. Data of patients discharged before 12 p.m. on the study days were excluded from the final analysis. Categorical data were analysed using Fisher's exact test or chi-squared test and are expressed as frequency. Continuous data were analysed using the Kruskal-Wallis test and are expressed as median (IQR). Multivariable regression models (generalized estimating equations), with a random effect for site, were used to evaluate variables associated with therapist-provided mobility events and outof-bed mobility (see Additional file 1$)$. Age $(<3$ or $\geq 3$ years) was explored as statistical interaction with covariates for the outcome of out-of-bed mobility. Adjusted odds ratios (aOR) are presented with 95\% confidence interval (CI). For the purpose of analysis, sites were categorized by European region using the definition in the end-of-life practices in intensive care units study (see Additional file 1, [19]. Two-tailed $P<0.05$ was considered statistically significant. IBM SPSS version 25.0 was used for all statistical analyses.

\section{Results \\ ICU characteristics}

A total of 38 PICUs from 15 countries participated, representing all European regions (see Additional file 1 eFigure1). Most were located in northern Europe (47\%) and were part of an academic teaching hospital (76\%), and half were paediatric medical-surgical-cardiac units (Additional file 1, eTable 1). The number of beds ranged from 4 to 31, with a median of 12 (IQR 9-17). Early mobilization protocols were available in $16 \%$ of the units. Dedicated PT or OT staff were present in $61 \%$ and $18 \%$ of the PICUs, respectively. In $87 \%$ (33/38), a prescription of a physician and/or nurse was required to consult a PT and/or OT.

\section{Patient baseline characteristics}

In total, 722 patients were screened for eligibility; 456 (63\%) patients were included (Additional file 1, eFigure 1). Fifty-three percent were boys, $70 \%$ were under the age of 3 years, and the median PICU length of stay on the study day was 14 days (IQR 6-36) (Table 1). Most were medical patients $(62 \%)$, and $59 \%$ of all included patients had surgery during the PICU admission. For $47 \%$ of all included patients, the PCPC prior to admission was $>2$, indicative of moderate or severe neurological/ cognitive disability.

\section{Patient clinical characteristics}

Two hundred and thirty-seven patients (52\%) were mechanically ventilated through an endotracheal tube or a tracheostomy during at the time of observation (Table 2). Half of the patients received at least one continuous sedative or analgesic infusion, mostly opioids (58\%) and benzodiazepines (43\%). The level of sedation was assessed in $73 \%(174 / 237)$ of the mechanically ventilated patients. Of the ventilated patients, 75\% (177/237) of the patients had a central venous catheter $(\mathrm{CVC})$ in place, and $51 \%(120 / 237)$ of these patients had a urinary catheter.

\section{Therapist-provided consultation and mobility}

The prevalence of PT- and/or OT-provided mobility was $39 \%$ (95\% CI 34.7-43.9\%) across the two study days. The prevalence significantly differed between the PICUs in the three European regions: northern, 36\%; central, 63\%; and southern, $25 \%(p<0.001)$. By $72 \mathrm{~h}$ of PICU admission, $24 \%$ of patients had received a PT and/or OT consultation, and $21 \%$ of those received mobilization 
Table 1 Patient baseline characteristics

\begin{tabular}{|c|c|c|c|c|}
\hline \multirow[t]{2}{*}{ Characteristics } & \multirow{2}{*}{$\begin{array}{l}\text { Patients } \\
\boldsymbol{N}=456\end{array}$} & \multirow{2}{*}{$\begin{array}{l}\text { PT/OT-provided mobility } \\
\mathbf{N}=179\end{array}$} & \multirow{2}{*}{$\begin{array}{l}\text { No PT/OT-provided mobility } \\
\mathbf{N}=277\end{array}$} & \multirow[t]{2}{*}{$\boldsymbol{p}$ value } \\
\hline & & & & \\
\hline Gender, female, $n(\%)$ & $213(46.7)$ & $88(49.2)$ & $125(45.1)$ & 0.442 \\
\hline Age, years, $n(\%)$ & & & & 0.033 \\
\hline $0-2$ & $319(70.0)$ & $111(62.0)$ & $208(75.1)$ & \\
\hline $3-6$ & $39(8.6)$ & $17(9.5)$ & $22(7.9)$ & \\
\hline $7-12$ & $54(11.8)$ & $27(15.1)$ & $27(9.7)$ & \\
\hline $13-18$ & $41(9.0)$ & $23(12.8)$ & $18(6.5)$ & \\
\hline$>18$ & $3(0.6)$ & $1(0.6)$ & $2(0.7)$ & \\
\hline Ethnicity, $n(\%)$ & & & & 0.3 \\
\hline White & $360(78.9)$ & $140(78.2)$ & $220(79.4)$ & \\
\hline Black & $27(5.9)$ & $8(4.5)$ & $19(6.9)$ & \\
\hline Asian & $43(9.4)$ & $17(9.5)$ & $26(9.4)$ & \\
\hline Hispanic & $4(0.8)$ & $2(1.1)$ & $2(0.7)$ & \\
\hline Other & $22(4.8)$ & $12(6.7)$ & $10(3.6)$ & \\
\hline Baseline PCPC, $n(\%)$ & & & & 0.031 \\
\hline 1: Good & $107(23.5)$ & $37(20.7)$ & $70(25.3)$ & \\
\hline 2: Mild disability & $124(26.1)$ & $37(20.7)$ & $82(29.6)$ & \\
\hline 3: Moderate disability & $96(21.1)$ & $43(24.0)$ & $53(19.1)$ & \\
\hline 4: Severe & $127(27.9)$ & $58(32.4)$ & $69(24.9)$ & \\
\hline 5: Coma/vegetative state & $7(1.5)$ & $4(2.2)$ & $3(1.1)$ & \\
\hline Reason of admission, $n(\%)$ & & & & 0.013 \\
\hline \multicolumn{5}{|l|}{ Medical } \\
\hline Respiratory & $125(27.4)$ & $49(27.4)$ & $76(27.4)$ & \\
\hline Cardiac & $61(13.4)$ & $17(9.5)$ & $44(15.9)$ & \\
\hline Haematology/oncology & $16(3.5)$ & $8(4.5)$ & $8(2.9)$ & \\
\hline Infectious/inflammatory & $21(4.6)$ & $13(7.3)$ & $8(2.9)$ & \\
\hline Neurologic & $36(7.9)$ & $13(7.3)$ & $23(8.3)$ & \\
\hline Other & $26(5.7)$ & $10(5.6)$ & $16(5.8)$ & \\
\hline \multicolumn{5}{|l|}{ Post-surgical } \\
\hline Cardiac & $81(17.8)$ & $27(15.1)$ & $54(19.5)$ & \\
\hline Neuro & $19(4.2)$ & $14(7.8)$ & $5(1.8)$ & \\
\hline Ortho & $3(0.7)$ & $1(0.6)$ & $2(0.7)$ & \\
\hline Other & $68(14.9)$ & $27(15.1)$ & $41(14.8)$ & \\
\hline Source of admission, $n$ (\%) & & & & 0.167 \\
\hline Emergency room & $55(12.1)$ & $17(9.5)$ & $38(13.7)$ & \\
\hline Floor/step down unit & $119(26.1)$ & $53(29.6)$ & $66(23.8)$ & \\
\hline Outside hospital & $155(34.0)$ & $56(31.3)$ & $99(35.7)$ & \\
\hline Operation room/post-anaesthesia & $69(15.1)$ & $34(19.0)$ & $35(12.6)$ & \\
\hline $\mathrm{NICU}$ & $39(8.6)$ & $11(6.1)$ & $28(10.1)$ & \\
\hline Home & $8(1.8)$ & $2(1.1)$ & $6(2.2)$ & \\
\hline Post-birth/delivery room & $9(1.8)$ & $4(2.2)$ & $4(1.4)$ & \\
\hline Other & $4(0.9)$ & $2(1.1)$ & $1(0.4)$ & \\
\hline $\mathrm{BMI}^{\mathrm{b}^{*}}$ & $15.1(12.8-17.9)$ & $15.6(13.3-18.5)$ & $14.7(12.3-17.6)$ & 0.002 \\
\hline Days of hospital stay at study day* & $22(\mathrm{IQR} 8-60)$ & $28(11-80)$ & $20(7-57)$ & 0.002 \\
\hline
\end{tabular}


Table 1 Patient baseline characteristics (Continued)

\begin{tabular}{lllll}
\hline Characteristics & Patients & PT/OT-provided mobility & No PT/OT-provided mobility & $\boldsymbol{p}$ value \\
& $\boldsymbol{N}=456$ & $\mathbf{N}=179$ & $\boldsymbol{N}=277$ \\
\hline Days of PICU stay at study day* & $14(6-36)$ & $17(8-45)$ & $12(6-32)$ & $8(4-22)$ \\
Postoperative day*1 & $11(4-31)$ & $18(7-39)$ & $164(59.2)$ & 0.002 \\
Surgery during PICU stay, yes (\%) & $278(58.9)$ & $107(59.8)$ & $29(42.0)$ & 0.001 \\
Ambulatory prior to admission if age $\geq 3$ years, no. (\%) & $63(46.0)$ & $34(50.0)$ & $35(12.6)$ & 0.393 \\
Unit mobility protocol, yes (\%) & $60(13.2)$ & $25(14.0)$ & & 0.673 \\
\hline
\end{tabular}

$P C P C$ paediatric cerebral performance category, $P T$ physical therapist, $O T$ occupational therapist

*Median (IQR)

${ }^{1}$ From most recent surgery

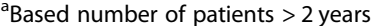

${ }^{\mathrm{b}}$ Missing data: BMI $(n=22)$

therapy. Still, $43 \%$ of patients, with a median PICU stay of 11 days (IQR 6-31), never received a PT or OT consultation. Receiving a PT and/or OT consultation was irrespective of the PCPC score (PCPC $\leq 222 \%$ vs. PCPC $\geq 3$ $26 \%, p=0.331$; Additional file 1, eFigure 3). Tables 1 and 2 display univariate analysis for demographic and clinical factors and the primary outcome of PT or OT provided mobility. We found that patients $\geq 3$ years of age received more frequently therapist-provided mobility compared with age $<3$ years, 35\% (111/319) versus 50\% (68/137) respectively $(p=0.003)$. Further, mechanically ventilated patients did not receive significantly more frequent therapistprovided mobility compared with those who were not $(42 \%$ vs. $36 \% ; p=0.181$ ). In total, 174 patient days included PTprovided mobility, and only ten patient days included OTprovided mobility. Therefore, we were not able to provide discipline-specific mobility associations.

In a logistic multivariable analysis adjusting for relevant demographic and clinical characteristics, therapistprovided mobility was associated with older age (aOR compared to age < 3: 7-12 years, 2.28 [95\% CI 1.23-4.22]) and moderate and severe baseline disability (PCPC 3 vs. PCPC 1: 2.12 [95\% CI 1.02-4.56] and PCPC 4 vs. PCPC1: 2.24 [95\% CI 1.14-4.40]). Therapist-provided mobility was also associated with having a CVC in place (aOR 1.63; 95\% CI 1.02-2.62) and with family presence during one of the study days (aOR 5.13; 95\% CI 2.55-10.32). In contrast, fewer therapist-provided mobility episodes were provided to children with urinary catheter in place (aOR 0.46; 95\% CI 0.22-0.92) (Additional file 1, eTable 2).

\section{All mobility events}

Mobilization events did not occur on 115 of 456 patient days $(25 \%)$, notably not in mechanically ventilated patients (75 of 115 patient days, 65\%). On the other 341 patient days, 1283 total mobility events occurred, with a median of 3 (IQR 3-5) per patient. In most cases $(n=$ $584,46 \%)$, a nurse alone provided the mobilization event; in $17 \%$ of cases, a nurse together with family; in $6 \%$ of cases, a nurse together with a PT or OT; and in
$16 \%$ of cases, family alone (Fig. 1). There were slight differences in mobilization between the European regions (Additional file 1, eFigure 4). Nurses mobilized critically ill children in the northern European countries less frequently (40\%) than in the southern (54\%) and central (51\%) countries.

Figure 2 shows the highest level of mobility achieved by patients on the study days. Among mechanically ventilated children, passive range of motion (age <3, 23\%; age $\geq 3,23 \%$ ) and being held by family or nurse (age $<3$, $27 \%$; age $\geq 3,22 \%$ ) were the most common mobility events. Among non-ventilated patients, being held by family or nurse was most frequent in children below age of 3 years (44\%), while bed-to-chair transfer was the commonest in over 2 -year-olds $(17 \%)$. Children $<3$ years were held by family or a nurse in $55 \%$ of central, $35 \%$ in northern, and $15 \%$ in southern European PICU (Additional file 1, eFigure 5).

\section{Out-of-bed mobility}

Out-of-bed mobility was achieved as the highest level of mobility for PICU patients on 248 study days, i.e. an overall prevalence of $46 \%$ (95\% CI 41.0-50.3\%). Seventyeight percent of the children below 3 years of age received out-of-bed mobility compared to $22 \%$ of patients 3 years of age and older $(p<0.001)$. Non-mechanically ventilated patients were significantly more likely than mechanically ventilated patients to achieve out-of-bed mobility ( $70 \%$ vs. $30 \% ; p<0.001)$. The most common out-of-bed activity for all patients was being held by a parent or a nurse $(n=519)$, with bed-to-chair transfer without standing $(n=120)$, and mat play $(n=51)$.

\section{Factors associated with out-of-bed mobility}

In a multivariable logistic regression model (Fig. 3, and Additional file 1, eTable 3), mechanical ventilation through an endotracheal tube (aOR $0.29,95 \%$ CI $0.12-$ 0.68 ), being admitted for a surgical reason (aOR 0.58, $95 \%$ CI $0.35-0.95)$, and the presence of a urinary catheter $(\mathrm{aOR} 0.39,95 \%$ CI $0.19-0.81)$ were negatively 
Table 2 Clinical characteristics of patients by PT/OT-provided mobility

\begin{tabular}{|c|c|c|c|c|}
\hline \multirow[t]{2}{*}{ Characteristics } & Patients & PT/OT-provided mobility & No PT/OT-provided mobility & \multirow[t]{2}{*}{$\boldsymbol{p}$ value } \\
\hline & $\boldsymbol{N}=456$ & $\boldsymbol{N}=179$ & $\boldsymbol{N}=277$ & \\
\hline Respiratory support, $n$ (\%) & & & & 0.445 \\
\hline No support & $67(14.7)$ & $28(15.6)$ & $39(14.1)$ & \\
\hline Nasal cannula or face mask & $30(6.6)$ & $12(6.7)$ & $18(6.5)$ & \\
\hline Heated high-flow nasal cannula & $50(11.0)$ & $15(8.4)$ & $35(12.6)$ & \\
\hline Trach collar & $18(3.9)$ & $5(2.8)$ & $13(4.7)$ & \\
\hline Non-invasive ventilation & $54(11.8)$ & 19 (10.6) & $35(12.6)$ & \\
\hline Mechanical ventilation via ETT & $178(39.0)$ & $71(39.7)$ & $107(38.6)$ & \\
\hline Mechanical ventilation via tracheostomy & $59(12.9)$ & $29(16.2)$ & $30(10.8)$ & \\
\hline $\mathrm{FiO2}^{*}$ & $30(25-45)$ & $35(25-45)$ & $30(25-40)$ & 0.091 \\
\hline Day of $\mathrm{MV}^{*}$ & $11(5-30)$ & $13(6-37)$ & $10(5-29)$ & 0.096 \\
\hline GCS* $^{*}$ & $14(9-15)$ & $13(9-15)$ & $14(9-15)$ & 0.470 \\
\hline Sedation score documented ${ }^{a}$, yes (\%) & $174(73.4)$ & $79(79.0)$ & $95(69.3)$ & 0.097 \\
\hline Sedatives/analgesics: $\geq 1$ continuous infusion, $n(\%)$ & $232(50.9)$ & $96(53.6)$ & $136(49.1)$ & 0.388 \\
\hline Vasoactive infusions, at least one, $n(\%)^{\mathrm{b}}$ & $50(11.0)$ & $19(10.6)$ & $31(11.2)$ & 0.879 \\
\hline Delirium screening performed & & & & 0.403 \\
\hline No, not available & $394(86.4)$ & $152(84.9)$ & $242(87.4)$ & \\
\hline Yes, positive screening & $5(1.1)$ & $1(0.6)$ & $4(1.4)$ & \\
\hline Yes, negative screening & $57(12.5)$ & $26(14.5)$ & $31(11.2)$ & \\
\hline Restraint, at least one, $n(\%)$ & $61(13.4)$ & $21(11.7)$ & $40(14.4)$ & 0.482 \\
\hline \multicolumn{5}{|l|}{ Devices, $n(\%)$} \\
\hline Endotracheal tube & $180(39.5)$ & $72(40.0)$ & $108(39.0)$ & 0.792 \\
\hline Tracheal cannula & $81(17.8)$ & $37(20.7)$ & $44(15.9)$ & 0.192 \\
\hline Central venous catheter & $290(63.6)$ & $118(65.9)$ & $172(62.1)$ & 0.427 \\
\hline Arterial line & $178(39.0)$ & $73(40.8)$ & $105(37.9)$ & 0.556 \\
\hline Haemodialysis catheter, $n$ (\%) & $21(4.6)$ & $7(3.9)$ & $14(5.1)$ & 0.652 \\
\hline ECMO cannula, $n(\%)$ & $14(3.1)$ & $4(2.2)$ & $10(3.6)$ & 0.580 \\
\hline Foley catheter, $n$ (\%) & $167(36.6)$ & $55(30.7)$ & $112(40.4)$ & 0.037 \\
\hline Surgical drain, $n(\%)$ & $40(8.8)$ & $14(7.8)$ & $26(9.4)$ & 0.614 \\
\hline Chest tube, $n(\%)$ & $39(8.3)$ & $14(7.8)$ & $25(9.0)$ & 0.773 \\
\hline Ventricular assist device, $n(\%)$ & $11(2.4)$ & $3(1.7)$ & $8(2.9)$ & 0.543 \\
\hline Intracranial pressure monitor, $n(\%)$ & $19(4.2)$ & $6(3.4)$ & $13(4.7)$ & 0.539 \\
\hline Pressure ulcer(s), at least one, $n(\%)$ & $48(10.5)$ & $15(8.4)$ & $33(11.9)$ & 0.275 \\
\hline Nurse to patient ratio, $n(\%)$ & & & & 0.503 \\
\hline $2: 1$ or $1: 1$ & $215(47.1)$ & $88(49.2)$ & $127(45.8)$ & \\
\hline $1: 2$ or $1: 3$ & $241(52.9)$ & $91(50.8)$ & $150(54.2)$ & \\
\hline Family present at bedside & $354(77.6)$ & $160(89.4)$ & $194(70.0)$ & $<0.001$ \\
\hline
\end{tabular}

PT physical therapist, OT occupational therapist, HFNC high-flow nasal cannula, CPAP continuous positive airway pressure, MV mechanical ventilation, ETT endotracheal tube, ECMO extracorporeal membrane oxygenation, CVC central venous catheter, FiO2 fractional inspired oxygen concentration, GCS Glasgow Coma Scale *Median (IQR)

alf mechanically ventilated (total, $n=237$; PT/OT provided, $n=100$; no PT/OT provided, $n=137$ )

${ }^{\mathrm{b}}$ Excluding milrinone

associated with out-of-bed mobility. Family presence had a strong positive association with out-of-bed mobility (aOR 7.83, 95\% CI 3.09-19.79).
Perceived barriers to mobilization

At least one barrier to mobilization was reported for 177 of 456 patients (39\%), with two or more barriers 


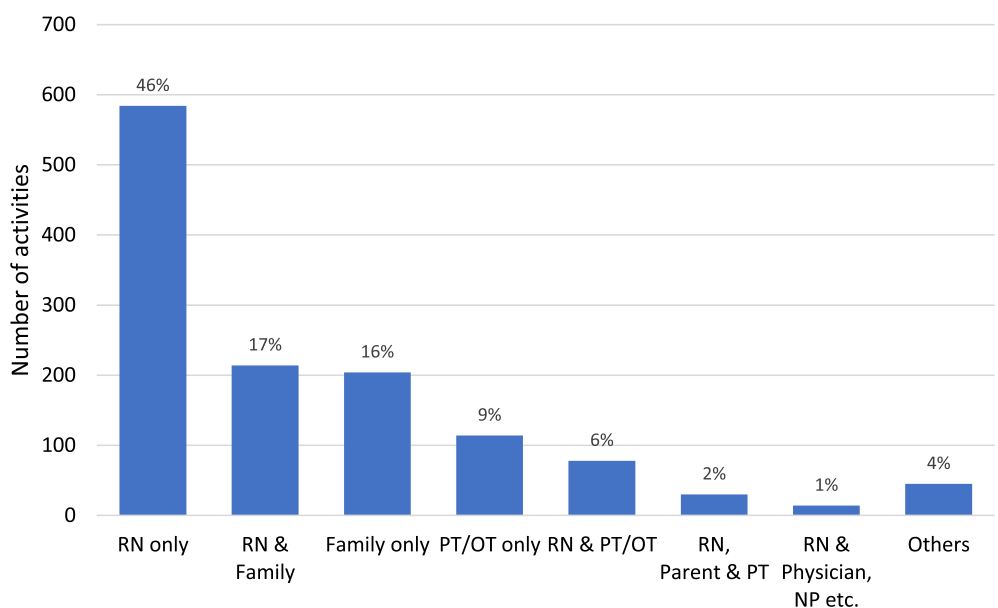

Fig. 1 Number of activities by clinician type. RN, registered nurse; PT, physical therapist; OT, occupational therapist; NP, nurse practitioner

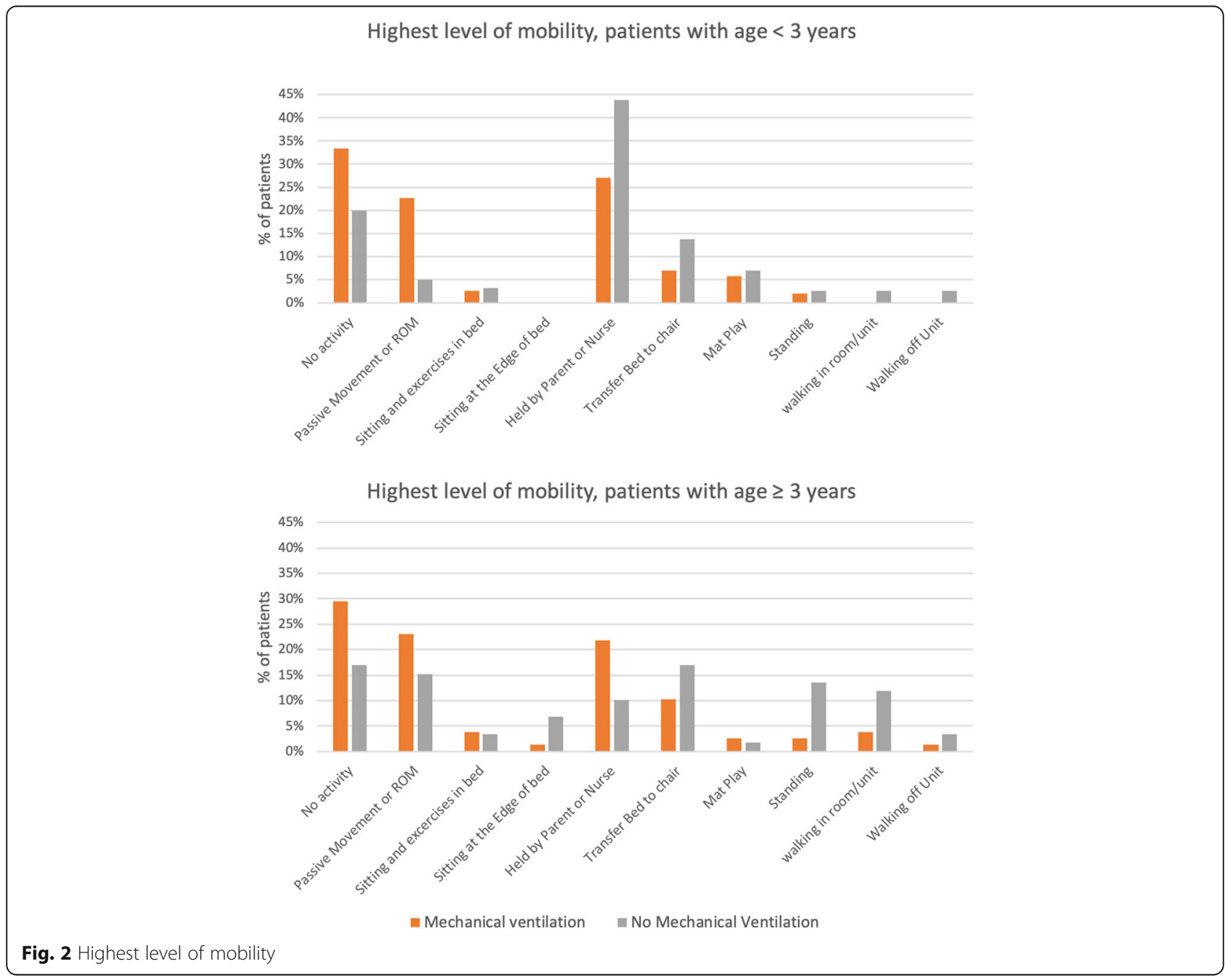




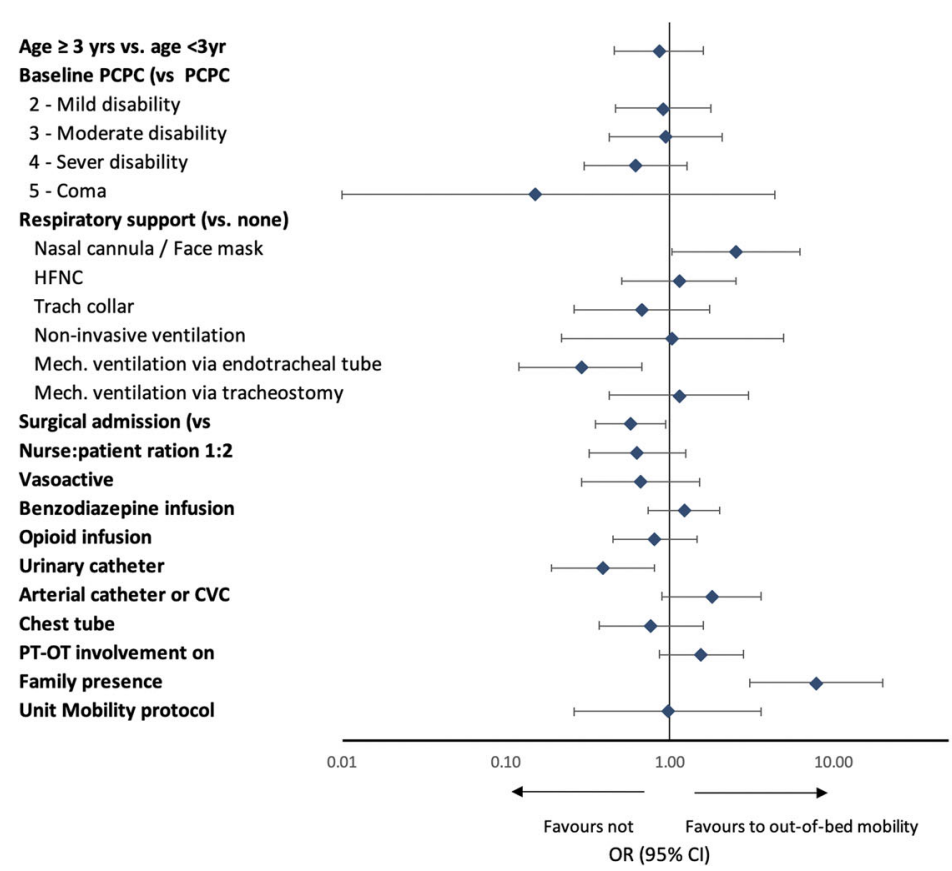

OR $(95 \% \mathrm{CI})$

$0.86(0.46-1.61)$

$0.91(0.47-1.77)$

$0.95(0.43-2.10)$

$0.62(0.30-1.27)$

$0.15(0.01-4.36)$

$2.55(1.04-6.28)$

$1.14(0.51-2.54)$

$0.67(0.26-1.75)$

$1.04(0.22-4.93)$

$0.29(0.12-0.68)$

$1.15(0.43-3.05)$

$0.58(0.35-0.95)$

$0.63(0.32-1.25)$

$0.66(0.29-1.52)$

$1.22(0.74-2.01)$

$0.81(0.45-1.46)$

$0.39(0.19-0.81)$

$1.80(0.90-3.60)$

$0.76(0.37-1.60)$

1.55 (0.86-2.81)

7.83 (3.09-19.79)

0.98 (0.26-3.62)

Fig. 3 Adjusted odds ratio for out-of-bed mobility on study day. The multivariable model included random effect for site, adjusted for admission reason, gender, and ethnicity in addition to all characteristics listed. Vasoactive infusion excluded milrinone. HFNC, high-flow nasal cannula; CPAP, continuous positive airway pressure; MV, mechanical ventilation; ETT, endotracheal tube; PCPC, paediatric cerebral performance category; CVC, central venous catheter; PT, physical therapist; OT, occupational therapist; EM, early mobilization

reported for 87 of these 177 patients (Additional file 1, eTable 4). Barriers were reported for $66 \%(76 / 115)$ of the patient days without mobilization activities. Barriers to mobilization were reported significantly more frequently for mechanically ventilated patient than for nonventilated patients $(49 \%$ vs. $28 \% ; p<0.001)$. The most common barriers were cardiovascular instability $(n=47$, $10 \%)$, oversedation $(n=39,9 \%)$, and medical contraindication $(n=37,8 \%)$.

\section{Potential adverse events}

Staff reported potential safety events in 74 (6\%) of 1286 mobility events in 43 patient days (9\%). These were more commonly among mechanically ventilated compared to non-ventilated patients ( $8 \%$ vs. $5 \% ; p=0.026)$. The most commonly reported potential safety events were decreased oxygen saturation $(n=33,3 \%)$, change in heart rate $(n=$ $25,2 \%)$, and change in blood pressure $(n=20,2 \%)$. An endotracheal tube dislocation occurred only once. There were no cardiac arrests or falls reported.

\section{Discussion}

This study presents the first estimates of routine mobilization practices in European PICUs. Mobilization did not occur on $25 \%$ of the study days, and when mobility did occur, it was facilitated mostly by nurses alone. Older children and children with severe disability most frequently received mobilization interventions provided by a physical therapist or occupational therapist with the help of nurses. One quarter of the patients were not mobilized at all due to cardiac instability, oversedation, or medical contraindication. We found that the rate of potential safety adverse events (6\%) was low relative to the large number of mobility events and similar to that reported in paediatric (4\%) and adult (3\%) studies [20]. Most of these potential safety events were transient vital sign changes. Only $0.2 \%$ of all mobility events were associated with dislodgement of a device, comparable to the $0.6 \%$ rate in adults [20]. Therefore, our data, in parallel with evidence from single-centre PICU studies [21, 22], suggest that mobilization of PICU patients is safe.

There are several important similarities and differences with the USA-PARK-PICU findings [12], a retrospective Canadian multicentre PICU study [23], and adult point prevalence studies of ICU mobilization practices [18]. Our observation of a $39 \%$ prevalence of therapistprovided mobility on the study days is consistent with the USA data (35\%) and the Canadian study (30\%) [23]. However, there was a large difference between the central European and northern and southern countries. A lower proportion of EU-PICUs (16\%) reported a unitbased mobility or rehabilitation protocol compared to the USA (27\%). The minority of units with systematic mobility approaches may in part explain the moderate 
prevalence of mobilization interventions provided by a physical therapist or occupational therapist. Integrating PTs and OTs into routine PICU care is integral to advancing infant and toddler's cognitive and physical development, especially important given that $70 \%$ of all PICU patients were < 3 years of age $[24,25]$. Notably, mechanically ventilated children were less likely to receive therapist-provided mobilization, in line with point prevalence studies in adults but different from USAPARK-PICU where there was no significant difference $[18,26-28]$.

Our study highlights the crucial role of nurses in mobilization of critically ill children. Nurses are a constant presence at the bedside, so it is not surprising that they provided the majority of mobilization events. In both the USA-PARK-PICU study and USA ARDS-Net point prevalence study in adults, nurses facilitated two thirds of mobility events $[12,18]$. PICU nurses understand the importance of early mobilization but may consider it as additional workload which may translate to lower prioritization [29]. A strong collaboration with therapists, unit-based protocols in place, and facilities and equipment for mobilization could facilitate the implementation of early mobilization in daily practice [30]. Although it would be a challenge for many PICUs with nursing shortages to accommodate such changes in practice, there may be a great benefit to patients with shorter PICU stays and duration of mechanical ventilation, less delirium, and reduced costs as has been observed in adults [31].

Presence of family was very strongly associated with increased out-of-bed mobility in our study. While the USA-PARK-PICU study observed that family presence was positively associated with mobility in children under 3, our European data magnifies this association powerfully. It is likely these family members provided assistance to facilitation success with the mobilization procedure. Parents indeed have a uniquely supportive role during physiotherapy that clinicians cannot provide [32, 33] which can help to decrease the child's anxiety and increase buy-in to participate [34]. With family visitation restrictions heightened during a global pandemic such as COVID-19, strategies are urgently needed to ensure that mobility is not negatively impacted.

Professional and organizational issues such as time constraints, lack of resources to implement early mobilization, and factors related with invasive devices and patient characteristics could be barriers to early rehabilitation across all ICU populations [35]. Our study show that decreased out-of-bed mobility of patients with an endotracheal tube and invasive devices in place is consistent with finding from adult studies and USAPICU data. However, a unique finding of the present study, similar to the USA, was that an indwelling urinary catheter was a barrier to out-of-bed mobility. Daily review of the potential for devices to be removed can both reduce the risk of hospital-related infections and avoid confining patients to bed. Out-of-bed mobility can be safe, when a device is secured during the pre-mobility planning [36], especially if a dedicated multi-professional mobilization protocol and trained team are available. Other perceived barriers to out-of-bed mobility included medical status, lack of physician order, isolation precautions, and oversedation. Having a mobilization protocol in place would, however, not be sufficient to overcome all these kinds of barriers. A culture change among PICU team is warranted.

Our study has several limitations. First, only PICUs with sufficient research staffing or interest in early mobilization may have participated, potentially biasing the results to overestimate mobilization practices. Still, this risk is low because only $16 \%$ of the participating PICUs had a formal protocol. Second, mobility assessments were unblinded, which may have led to greater mobility delivery on the study days. Efforts had been made to limit knowledge of the study, and the observed relatively low prevalence makes it unlikely that singleday escalation of mobility efforts has biased the overall estimates. Third, we were not able to report whether a patient was medically able to be mobilized or get out of bed. Fourth, combining PT- and OT-provided mobility as the primary outcome may not recognize their unique contributions. But, due to small numbers of OTprovided mobility, we were not able to provide discipline-specific mobility associations. Further, in lack of knowing the proportion of PTs/OTs who work exclusively in the PICU, we could not analyse the difference in therapist-provided mobility between those who work exclusively in the PICU and those who not. Finally, data of non-participating PICUs were not available, thereby limiting the generalizability of the results. Although a remarkable number of 38 PICUs from 16 European countries was involved in this study, the number of participating PICUs per country differed greatly, with the strongest representation of PICUs from Northern Europe. In view of the regional differences, the results of this study should be interpreted with caution. Further, France, one of the biggest European countries with many PICUs, was not represented in this study. There may be additional cultural differences in PICU practices across Europe, and the study findings cannot be considered a true representation of all PICUs in Europe.

\section{Conclusions}

In this point prevalence study, $25 \%$ of critically ill children across Europe were completely immobile. When mobilization occurred, the most common activity was children being held by family or nurses. Nurses are the 
most frequent providers of mobilization and therapists are less frequently consulted, bringing to light differences between European regions and highlighting the need for a systematic, interprofessional approach to mobilization across PICUs. Removing modifiable barriers such as oversedation and lack of medical order, combined with facilitation of parental involvement, will be important to increase mobilization and rehabilitation in European PICUs, especially in ventilated children. Finally, the short- and longterm impacts of early mobilization and rehabilitation programmes should be evaluated in critically ill children to determine best practice for paediatric critical care.

\section{Supplementary information}

Supplementary information accompanies this paper at https://doi.org/10. 1186/s13054-020-02988-2.

Additional file 1. Mobilisation practices in critically ill children: A European point prevalence study (EU PARK-PICU), contain the following information: 1 Additional description of data analysis. 2 eTables. 3 eFigures.

\section{Abbreviations}

PICU: Paediatric intensive care unit; PCPC: Paediatric Cerebral Performance Score; PT: Physical therapy; OT: Occupational therapy

\section{Acknowledgements}

PARK-PICU EU Collaborators: Paul C. Ritson MCSP, Alder Hey Children's NHS Foundation Trust, Liverpool UK; Filippia Nikolaou MD, Children Hospital 'P \& A' Aglaia Kyriakou, Athens Greece; Marjorie de Neef RN MSc, Amsterdam UMC, Emma Children's Hospital, Amsterdam, The Netherlands; Martin Kneyber MD PhD, Beatrix Children's Hospital, Groningen, The Netherlands; Kate Penny-Thomas RM, Birmingham Women \& Children's Hospital, Birmingham, UK; Christina Linton, Bristol Royal Hospital for Children, Bristol UK; Reinis Balmaks MD, Children's Clinical University Hospital, Riga, Latvia; Matthias Richter MD, Universitätsklinikum Carl Gustav Carus Klinik für Kinder- und Jugendmedizin, Dresden, Germany; Fabrizio Chiusolo MD, Children's Hospital Bambino Gesù - PICU, Rome, Italy; Corrado Cecchetti MD, Children's Hospital Bambino Gesù - Emergency Department PICU, Rome, Italy; Marco Roberti MSc RN, Children's Hospital Bambino Gesù -CICU, Rome, Italy; Michela Di Furia MSc RN, Children's Hospital Bambino Gesù - Palidoro, Italy; Chantal Grandjean RN MSc, Lausanne University Hospital (CHUV), Lausanne, Switzerland; Bettina Nygaard RN PhD, Copenhagen University Hospital, Rigshospitalet, Copenhagen, Denmark; Yolanda Lopez MD, Cruces University Hospital, Bilbao, Spain; Tolga Koroglu MD十, Dokuz Eylül University, Izmir, Turkey; Tolga Besci MD, Dokuz Eylül University, Izmir, Turkey; Roberta Da Rin Della Mora RN PhD, IRCCS Istituto Giannina Gaslini, Genova, Italy; Rachel S Agbeko MD PhD, Great North Children's Hospital, Newcastle upon Tyne, UK; Emma Borrows MD, Great Ormond Street Hospital, London, UK; Nathalie Bochaton MSc, Geneva University Hospital (HUG), Geneva, Switzerland; Janet Mattsson RN PhD, Karolinska, Stockholm, Sweden; Anne Ksellmann MD, Kinderherzzentrum Sankt Augustin, St Augustin, Germany; Barbara Hero RN, Children's University Hospital Cologne, Köln, Germany; Jowita Rosada-Kurasinska, Department of Pediatric Anesthesiology and Intensive Therapy, Poznań University of Medical Sciences, Poznań, Poland; Magdalena Świder MD, Kliniczny Szpital Wojewódzki No 2 w Rzeszowie, Rzeszów, Poland; Amabile Bonaldi RN, Ospedale Maggiore, Verona, Italy; Cristina Giugni MD, Meyer Children's Hospital, Florence, Italy; Siva Oruganti MD, Noah's Ark Children's Hospital for Wales, Cardiff, UK; Simon Gates PT MSc, Nottingham Children's Hospital and Neonatology, Nottingham University Hospitals NHS Trust, Nottingham, England; Hazel Smith RM PhD, Children's Health Ireland at Crumlin, Dublin, Ireland; Annelies van Zwol MD PhD, Radboudumc-Amila Children's Hospital, Nijmegen, The Netherlands; Jenna Hills PT, Royal Hospital for Children, Glasgow, Scotland; Johanna Conroy PT, Royal Manchester Children's Hospital, Manchester, England; Mark Bebbington MD, Royal Stoke University Hospital, Stoke-on-Trent, UK; Felix Neunhoeffer MD PhD, Department of Pediatric
Cardiology, Pulmonology and Pediatric Intensive Care Medicine, University Children's Hospital Tübingen, Tübingen, Germany; Els Duval MD PhD, UZA Antwerp, Belgium.

Ko Hagoort MA, Erasmus MC, for editing the manuscript.

\section{Authors' contributions}

El and SK designed the study. El, BS, JM, IH, OG, AB, and AR coordinated the study. El analysed the data. El and SK interpreted the results. El and SK wrote the manuscript. All authors contributed to the drafting of the manuscript and critical revision of it for important intellectual content and approved the final version of the manuscript. El had full access to all the data in the study and takes responsibility for the integrity of the data and the accuracy of the data analysis. All authors read and approved the final manuscript.

\section{Funding}

This research received no specific funding.

\section{Availability of data and materials}

The datasets used and/or analysed during the current study are available from the corresponding author on reasonable request.

\section{Ethics approval and consent to participate}

This study was approved by the institutional review board of the Erasmus Medical Center (EMC-2017-1037) and waived the need for an informed consent.

\section{Consent for publication}

Not applicable

\section{Competing interests}

All authors declare that they have no conflict of interest. All authors have completed and submitted the International Committee of Medical Journal Editors (ICMJE) form for disclosure of potential conflicts of interest.

\section{Author details}

${ }^{1}$ Pediatric Intensive Care Unit, Department of Paediatric Surgery, Erasmus Medical Center - Sophia Children's Hospital, P.O. Box 2060, 3000 CB Rotterdam, The Netherlands. ${ }^{2}$ Nursing Science, Department of Internal Medicine, Erasmus Medical Center, Rotterdam, The Netherlands. ${ }^{3}$ Birmingham Acute Care Research Group, Institute of Inflammation and Ageing, University of Birmingham, Birmingham, UK. ${ }^{4}$ Paediatric Intensive Care Unit, Birmingham Women \& Children's Hospital, Birmingham, UK. ${ }^{5}$ Nottingham Children's Hospital \& Neonatology, Nottingham University Hospitals NHS Trust, Nottingham, UK. ${ }^{6}$ School of Health Sciences, The University of Nottingham, Nottingham, UK. ${ }^{7}$ Pediatric Intensive Care Unit, Universitätsmedizin Mainz, Zentrum für Kinder- und Jugendmedizin, Mainz, Germany. ${ }^{8}$ Healthcare professional development Unit, Bambino Gesù Children's Hospital, Rome, Italy. 'Department of Pediatric Anesthesiology and Intensive Therapy, Poznań University of Medical Sciences, Poznań, Poland. ${ }^{10}$ Institute of Higher Education and Research in Healthcare, University of Lausanne and Lausanne University Hospital, Lausanne, Switzerland.

${ }^{11}$ Department of Anesthesiology and Critical Care Medicine, Johns Hopkins University School of Medicine, Baltimore, MD, USA. ${ }^{12}$ Department of Pediatrics, Johns Hopkins University School of Medicine, Baltimore, MD, USA. ${ }^{13}$ Department of Physical Medicine \& Rehabilitation, Johns Hopkins University School of Medicine, Baltimore, MD, USA. ${ }^{14}$ Alder Hey Children's NHS Foundation Trust, Liverpool, UK. ${ }^{15}$ Children Hospital 'P \& A 'Aglaia Kyriakou, Athens, Greece. ${ }^{16}$ Amsterdam UMC-Emma Children's Hospital, Amsterdam, The Netherlands. ${ }^{17}$ University Medical Center Groningen, Beatrix Children's Hospital, Groningen, The Netherlands. ${ }^{18}$ Birmingham Women \& Children's Hospital, Birmingham, UK. ${ }^{19}$ Bristol Royal Hospital for Children, Bristol, UK. ${ }^{20}$ Children's Clinical University Hospital, Riga, Latvia. ${ }^{21}$ Universitätsklinikum Carl Gustav Carus Klinik für Kinder- und Jugendmedizin, Dresden, Germany. ${ }^{22}$ Children's Hospital Bambino Gesù - PICU, Rome, Italy. ${ }^{23}$ Children's Hospital Bambino Gesù - Emergency Department PICU, Rome, Italy. ${ }^{24}$ Children's Hospital Bambino Gesù -CICU, Rome, Italy. ${ }^{25}$ Children's Hospital Bambino Gesù, Palidoro, Italy. ${ }^{26}$ Lausanne University Hospital (CHUV), Lausanne, Switzerland. ${ }^{27}$ Copenhagen University Hospital, Rigshospitalet, Copenhagen, Denmark. ${ }^{28}$ Cruces University Hospital, Bilbao, Spain. ${ }^{29}$ Dokuz Eylül University, Izmir, Turkey. ${ }^{30}$ IRCCS Istituto Giannina Gaslini, Genova, Italy. ${ }^{31}$ Great North Children's Hospital, Newcastle upon Tyne, UK. ${ }^{32}$ Great Ormond Street 
Hospital, London, UK. ${ }^{33}$ Geneva University Hospital (HUG), Geneva, Switzerland. ${ }^{34}$ Karolinska, Stockholm, Sweden. ${ }^{35}$ Kinderherzzentrum Sankt Augustin, St Augustin, Germany. ${ }^{36}$ Children's University Hospital Cologne, Köln, Germany. ${ }^{37}$ Kliniczny Szpital Wojewódzki No 2 w Rzeszowie, Rzeszów, Poland. ${ }^{38}$ Ospedale Maggiore, Verona, Italy. ${ }^{39}$ Meyer Children's Hospital, Florence, Italy. ${ }^{40}$ Noah's Ark Children's Hospital for Wales, Cardiff, UK. ${ }^{41}$ Nottingham Children's Hospital and Neonatology, Nottingham University Hospitals NHS Trust, Nottingham, England. ${ }^{42}$ Children's Health Ireland at Crumlin, Dublin, Ireland. ${ }^{43}$ Radboudumc-Amila Children's Hospital, Nijmegen, The Netherlands. ${ }^{44}$ Royal Hospital for Children, Glasgow, Scotland. ${ }^{45}$ Royal Manchester Children's Hospital, Manchester, England. ${ }^{46}$ Royal Stoke University Hospital, Stoke-on-Trent, UK. ${ }^{47}$ Department of Pediatric Cardiology, Pulmonology and Pediatric Intensive Care Medicine, University Children's Hospital Tübingen, Tübingen, Germany. ${ }^{48}$ UZA Antwerp, Edegem, Belgium.

Received: 30 January 2020 Accepted: 12 May 2020

Published online: 24 June 2020

\section{References}

1. Kudchadkar SR, Yaster M, Punjabi NM. Sedation, sleep promotion, and delirium screening practices in the care of mechanically ventilated children: a wake-up call for the pediatric critical care community. Crit Care Med. 2014;42(7):1592-600.

2. Field-Ridley A, Dharmar M, Steinhorn D, McDonald C, Marcin JP. ICUacquired weakness is associated with differences in clinical outcomes in critically ill children. Pediatr Crit Care Med. 2016;17(1):53-7.

3. Dantas CM, Silva PF, Siqueira FH, Pinto RM, Matias S, Maciel C, Oliveira MC, Albuquerque CG, Andrade FM, Ramos FF, et al. Influence of early mobilization on respiratory and peripheral muscle strength in critically ill patients Influencia da mobilizacao precoce na forca muscular periferica e respiratoria em pacientes criticos. Rev Bras Ter Intensiva. 2012;24(2):173-8.

4. Curley MA, Quigley SM, Lin M. Pressure ulcers in pediatric intensive care: incidence and associated factors. Pediatr Crit Care Med. 2003;4(3):284-90.

5. Manning MJ, Gauvreau K, Curley MA. Factors associated with occipital pressure ulcers in hospitalized infants and children. Am J Crit Care. 2015; 24(4):342-8.

6. Schweickert WD, Pohlman MC, Pohlman AS, Nigos C, Pawlik AJ, Esbrook CL, Spears L, Miller M, Franczyk M, Deprizio D, et al. Early physical and occupational therapy in mechanically ventilated, critically ill patients: a randomised controlled trial. Lancet. 2009;373(9678):1874-82.

7. Knoester $\mathrm{H}$, Bronner MB, Bos AP. Surviving pediatric intensive care: physical outcome after 3 months. Intensive Care Med. 2008;34(6):1076-82.

8. Rennick JE, Rashotte J. Psychological outcomes in children following pediatric intensive care unit hospitalization: a systematic review of the research. J Child Health Care. 2009;13(2):128-49.

9. Choong K, Fraser D, Al-Harbi S, Borham A, Cameron J, Cameron S, Cheng J, Clark H, Doherty T, Fayed N, et al. Functional recovery in critically ill children, the "WeeCover" multicenter study. Pediatr Crit Care Med. 2018;19(2):145-54.

10. Schweickert WD, Kress JP. Implementing early mobilization interventions in mechanically ventilated patients in the ICU. Chest. 2011;140(6):1612-7.

11. Wieczorek B, Ascenzi J, Kim Y, Lenker H, Potter C, Shata NJ, Mitchell L, Haut C, Berkowitz I, Pidcock F, et al. PICU Up!: impact of a quality improvement intervention to promote early mobilization in critically ill children. Pediatr Crit Care Med. 2016;17(12):e559-66.

12. Kudchadkar SR, Nelliot A, Awojoodu R, Vaidya D, Traube C, Walker T, Needham DM, Prevalence of Acute Rehabilitation for Kids in the PI, the Pediatric Acute Lung I, Sepsis Investigators N et al: Physical Rehabilitation in Critically III Children: A Multicenter Point Prevalence Study in the United States. Crit Care Med. 2020;48(5):634-44.

13. Parry SM, El-Ansary D, Cartwright MS, Sarwal A, Berney S, Koopman R, Annoni R, Puthucheary Z, Gordon IR, Morris PE, et al. Ultrasonography in the intensive care setting can be used to detect changes in the quality and quantity of muscle and is related to muscle strength and function. J Crit Care. 2015;30(5):1151 e1159-14.

14. Cuello-Garcia CA, Mai SHC, Simpson R, Al-Harbi S, Choong K. Early mobilization in critically ill children: a systematic review. J Pediatr. 2018;203: 25-33 e26.

15. Patel BK, Pohlman AS, Hall JB, Kress JP. Impact of early mobilization on glycemic control and ICU-acquired weakness in critically ill patients who are mechanically ventilated. Chest. 2014;146(3):583-9.
16. PARK-PICU Data Collection Forms. https:/park.web.jhu.edu/data-collection-forms/. Accessed 28 Jan 2020.

17. Fiser DH, Tilford JM, Roberson PK. Relationship of illness severity and length of stay to functional outcomes in the pediatric intensive care unit: a multiinstitutional study. Crit Care Med. 2000;28(4):1173-9.

18. Jolley SE, Moss M, Needham DM, Caldwell E, Morris PE, Miller RR, Ringwood $\mathrm{N}$, Anders M, Koo KK, Gundel SE, et al. Point prevalence study of mobilization practices for acute respiratory failure patients in the United States. Crit Care Med. 2017;45(2):205-15

19. Sprung CL, Cohen SL, Sjokvist P, Baras M, Bulow HH, Hovilehto S, Ledoux D, Lippert A, Maia P, Phelan D, et al. End-of-life practices in European intensive care units: the Ethicus Study. JAMA. 2003;290(6):790-7.

20. Nydahl P, Sricharoenchai T, Chandra S, Kundt FS, Huang M, Fischill M, Needham DM. Safety of patient mobilization and rehabilitation in the intensive care unit. Systematic review with meta-analysis. Ann Am Thorac Soc. 2017;14(5):766-77.

21. Fink EL, Beers SR, Houtrow AJ, Richichi R, Burns C, Doughty L, Ortiz-Aguayo R, Madurski CA, Valenta C, Chrisman M, et al. Early protocolized versus usual care rehabilitation for pediatric neurocritical care patients: a randomized controlled trial. Pediatr Crit Care Med. 2019;20(6):540-50.

22. Wieczorek B, Burke C, Al-Harbi A, Kudchadkar SR. Early mobilization in the pediatric intensive care unit: a systematic review. J Pediatr Intensive Care. 2015:2015:129-70

23. Choong K, Foster G, Fraser DD, Hutchison JS, Joffe AR, Jouvet PA, Menon K, Pullenayegum E, Ward RE, Canadian Critical Care Trials G. Acute rehabilitation practices in critically ill children: a multicenter study. Pediatr Crit Care Med. 2014;15(6):e270-9.

24. Ross K, Heiny E, Conner S, Spener P, Pineda R. Occupational therapy, physical therapy and speech-language pathology in the neonatal intensive care unit: patterns of therapy usage in a level IV NICU. Res Dev Disabil. 2017:64:108-17.

25. Case-Smith J, Rogers S. Physical and occupational therapy. Child Adolesc Psychiatr Clin N Am. 1999;8(2):323-45.

26. Berney SC, Harrold M, Webb SA, Seppelt I, Patman S, Thomas PJ, Denehy L. Intensive care unit mobility practices in Australia and New Zealand: a point prevalence study. Crit Care Resusc. 2013;15(4):260-5.

27. Nydahl P, Ruhl AP, Bartoszek G, Dubb R, Filipovic S, Flohr HJ, Kaltwasser A, Mende $\mathrm{H}$, Rothaug $\mathrm{O}$, Schuchhardt $\mathrm{D}$, et al. Early mobilization of mechanically ventilated patients: a 1-day point-prevalence study in Germany. Crit Care Med. 2014;42(5):1178-86.

28. Sibilla A, Nydahl P, Greco N, Mungo G, Ott N, Unger I, Rezek S, Gemperle S, Needham DM, Kudchadkar SR. Mobilization of mechanically ventilated patients in Switzerland. J Intensive Care Med. 2020;35(1):55-62.

29. Zheng K, Sarti A, Boles S, Cameron S, Carlisi R, Clark H, Khawaji A, Awladthani S, Al-Harbi S, Choong K. Impressions of early mobilization of critically ill children-clinician, patient, and family perspectives. Pediatr Crit Care Med. 2018;19(7):e350-7.

30. Hopkins RO, Choong K, Zebuhr CA, Kudchadkar SR. Transforming PICU culture to facilitate early rehabilitation. J Pediatr Intensive Care. 2015:4(4):204-11.

31. Pun BT, Balas MC, Barnes-Daly MA, Thompson JL, Aldrich JM, Barr J, Byrum D, Carson SS, Devlin JW, Engel HJ, et al. Caring for critically ill patients with the ABCDEF bundle: results of the ICU liberation collaborative in over 15,000 adults. Crit Care Med. 2019;47(1):3-14.

32. Sottile PD, Nordon-Craft A, Malone D, Schenkman M, Moss M. Patient and family perceptions of physical therapy in the medical intensive care unit. Crit Care. 2015;30(5):891-5.

33. Parisien RB, Gillanders K, Hennessy EK, Herterich L, Saunders K, Lati J, Dos Santos S, Hassall A, O'Brien KK. Experiences of four parents with physical therapy and early mobility of their children in a pediatric critical care unit: a case series. J Pediatr Rehabil Med. 2016;9(2):159-68.

34. Bastani F, Abadi TA, Haghani $\mathrm{H}$. Effect of family-centered care on improving parental satisfaction and reducing readmission among premature infants: a randomized controlled trial. J Clin Diagn Res. 2015;9(1):SC04-8.

35. Dubb R, Nydahl P, Hermes C, Schwabbauer N, Toonstra A, Parker AM, Kaltwasser A, Needham DM. Barriers and strategies for early mobilization of patients in intensive care units. Ann Am Thorac Soc. 2016;13(5):724-30.

36. Chohan S, Ash S, Senior L. A team approach to the introduction of safe early mobilisation in an adult critical care unit. BMJ Open Qual. 2018;7(4):e000339.

\section{Publisher's Note}

Springer Nature remains neutral with regard to jurisdictional claims in published maps and institutional affiliations. 